\title{
Existence of a Photonic Gap in Periodic Dielectric Structures
}

\author{
K. M. Ho, C. T. Chan, and C. M. Soukoulis \\ Ames Laboratory and Department of Physics, Iowa State University, Ames, Iowa 50011
}

(Received 4 September 1990)

\begin{abstract}
Using a plane-wave expansion method, we have solved Maxwell's equations for the propagation of electromagnetic waves in a periodic lattice of dielectric spheres (dielectric constant $\epsilon_{a}$ ) in a uniform dielectric background $\left(\epsilon_{b}\right)$. Contrary to experiment, we find that fcc dielectric structures do not have a "photonic band gap" that extends throughout the Brillouin zone. However, we have determined that dielectric spheres arranged in the diamond structure do possess a full photonic band gap. This gap exists for refractive-index contrasts as low as 2 .
\end{abstract}

PACS numbers: 71.25.Cx, 41.10.Hv, 84.90.+a

Recently, there has been growing interest in studies of the propagation of electromagnetic (EM) waves in disordered and/or periodic dielectric structures. ${ }^{1}$ This interest is partly due to the possibility of the observation of Anderson localization ${ }^{2}$ of EM waves in disordered dielectric structures ${ }^{3-7}$ and also to the possible existence of photonic band gaps in 3D periodic dielectric structures. ${ }^{8-13}$ In analogy to the case of electron waves propagating in a crystal, light waves traveling in periodic structures will be described in terms of photonic bands with the possibility of the existence of frequency gaps where the propagation of EM waves is forbidden. In the original proposal for photonic band structure, ${ }^{10}$ it was suggested that the inhibition of spontaneous emission in such gaps can be utilized to substantially enhance the performance of semiconductor lasers and other quantum electronic devices. It has also been speculated ${ }^{14}$ that the absence of vacuum fluctuations in the photonic gap will lead to new physical phenomena.

For the case of EM-wave localization, there exists as yet $^{3-7}$ no proof of the existence of classical wave localization in a system of disordered dielectric structures. Drake and Genack ${ }^{15}$ have recently reported measurements of the optical diffusion coefficient ${ }^{7}$ in a system of close-packed titania spheres, strongly suggesting that the critical regime very close to localization has been reached. In addition, John ${ }^{8}$ has proposed that optical localization for EM waves near a photonic band gap might be achieved by weak disordering of a periodic arrangement of spheres. These recent developments suggest that a clear experimental demonstration of optical localization is imminent.

It is therefore very important to obtain a structure with a frequency gap where the propagation of EM waves is forbidden for all wave vectors. Investigations in this field are only just beginning. In the first experiment on photonic band structure, Yablonovitch and Gmitter ${ }^{11}$ reported a photonic gap in a face-centered-cubic (fcc) dielectric structure. On the theoretical side, studies of the propagation of EM waves have so far been based upon the scalar-wave approximation ${ }^{8,9,12,13}$ in which the vector nature of the EM field is ignored. This approximation gives qualitatively incorrect results for the existence of photonic gaps (see discussion below). Results for the vector EM field have only been obtained for a system of random dielectric spheres within the coherent potential approximation. ${ }^{3-7}$

In this paper we report calculations for the photonic band structure of periodic arrangements of dielectric spheres in the fcc and the diamond structures. Expanding the EM fields with a plane-wave basis set, we solve Maxwell's equation exactly, taking the vector nature of the EM field fully into account. Comparison of our calculational results of the fcc structure with experiment indicates that while the experimental data and theory agree very well over most of the Brillouin zone, there are two symmetry points ( $W$ and $U$ ) where the experiment indicates a gap while calculations show that propagating modes exist. We believe the fcc structure exhibits a pseudogap rather than a full photonic band gap; that is, there is a region where a band gap exists over most, but not all, of the Brillouin zone, resulting in a region of low density of states rather than a forbidden frequency gap. On the other hand, we found that the diamond dielectric structure does possess a full photonic band gap. Details of the calculations and discussion of the results will be presented in the remainder of the paper.

In a periodic structure where the dielectric constant $\epsilon(\mathbf{r})$ is position dependent, Maxwell's equations for EM waves can be written as

$$
\nabla \times \mathbf{E}=i(\omega / c) \mathbf{H}, \quad \nabla \times \mathbf{H}=-i(\omega / c) \epsilon(\mathbf{r}) \mathbf{E},
$$

which can be further simplified to

$$
\boldsymbol{\nabla} \times\left(\frac{1}{\epsilon(\mathbf{r})} \nabla \times \mathbf{H}\right)=\frac{\omega^{2}}{c^{2}} \mathbf{H} .
$$

Since $\epsilon(\mathbf{r})$ is periodic, we can use Bloch's theorem to expand the $\mathbf{H}$ field in plane waves,

$$
\mathbf{H}(\mathbf{r})=\sum_{\mathbf{G}} \sum_{\lambda=1}^{2} h_{\mathbf{G}, \lambda} \hat{\mathbf{e}}_{\lambda} e^{i(\mathbf{k}+\mathbf{G}) \cdot \mathbf{r}},
$$


where $\mathbf{k}$ is a wave vector in the Brillouin zone of the lattice, $\mathbf{G}$ is a reciprocal-lattice vector, and $\hat{\mathbf{e}}_{1}, \hat{\mathbf{e}}_{2}$ are unit vectors perpendicular to $\mathbf{k}+\mathbf{G}$ because of the transverse nature of $\mathbf{H}$ (i.e., $\boldsymbol{\nabla} \cdot \mathbf{H}=0$ ). Substituting into Eq. (2) we obtain the following matrix equations:

$$
\sum_{\mathbf{G}^{\prime}, \lambda^{\prime}} H_{\mathbf{G}, \mathbf{G}^{\prime}}^{\lambda, \lambda_{\mathbf{G}^{\prime}, \lambda^{\prime}}}=\left(\omega^{2} / c^{2}\right) h_{\mathbf{G}, \lambda}
$$

where

$H_{\mathbf{G}, \mathbf{G}^{\prime}}=|\mathbf{k}+\mathbf{G}|\left|\mathbf{k}+\mathbf{G}^{\prime}\right| \epsilon_{\mathbf{G}, \mathbf{G}^{\prime}}^{-1}\left(\begin{array}{cc}\hat{\mathbf{e}}_{2} \cdot \hat{\mathbf{e}}_{2^{\prime}} & -\hat{\mathbf{e}}_{2} \cdot \hat{\mathbf{e}}_{1^{\prime}} \\ -\hat{\mathbf{e}}_{1} \cdot \hat{\mathbf{e}}_{2^{\prime}} & \hat{\mathbf{e}}_{1} \cdot \hat{\mathbf{e}}_{1^{\prime}}\end{array}\right)$

and $\epsilon_{\mathbf{G}, \mathbf{G}^{\prime}}=\epsilon\left(\mathbf{G}-\mathbf{G}^{\prime}\right)$ is the Fourier transform of $\epsilon(\mathbf{r})$.

Equation (4) can be solved using standard matrixdiagonalization methods. ${ }^{16}$ We note that in our method the structure of the dielectric crystal enters the calculation only through the position-dependent dielectric function $\epsilon(\mathbf{r})$ which is evaluated on a fine grid in the realspace unit cell and Fourier transformed into reciprocal space. Thus our method is able to treat exactly any periodic arrangements of objects with arbitrary shapes and filling ratios. We find that the results for the lowest ten bands converge fairly rapidly. Results reported in this paper were obtained with matrix sizes of the order of 750. Frequencies are converged to better than $1 \%$.

We performed numerical calculations for the fcc lattices for cases of both dielectric spheres and air spheres. Although an extensive search was made with various refractive-index contrasts and filling ratios $(f)$, none of the fcc structures we investigated exhibit a complete photonic band gap. On the whole, there is good, though not perfect, agreement with the available experimental data. "For example, an investigation of the dependence of the band gaps at the $X$ point and $L$ point as a function of filling ratio $(f)$ for the case of air spheres in a $n=3.5$ dielectric gives the right trends for the variation of the $L$ and $X$ gaps with filling ratio, with the $X$ gap becoming zero at $f=67 \%$.

Experimentally, ${ }^{11}$ the best-performing structure was a fcc dielectric lattice of air spheres in a material with refractive index of 3.5 at a filling ratio of $14 \%$ dielectric material and $86 \%$ air. In this configuration, the filling ratio of the spherical air atoms is higher than the closepacking density (74\% for the fcc lattice) and the spheres overlap slightly. However, this does not cause any problem in our calculational scheme, since $\epsilon(\mathbf{r})$ is represented numerically on a fine grid in real space. (The filling ratio of the structure is obtained from numerical integration over the real-space grid.) We plot in Fig. 1 our calculated photon bands for this structure along important symmetry lines in the Brillouin zone (BZ). Our results are similar to the experimental reported band structure for most of the wave vectors. However, there are serious disagreements near the symmetry points $W$ and $U$ : At the $W$ point we found the second and third bands to be degenerate, independent of refractive-index contrasts

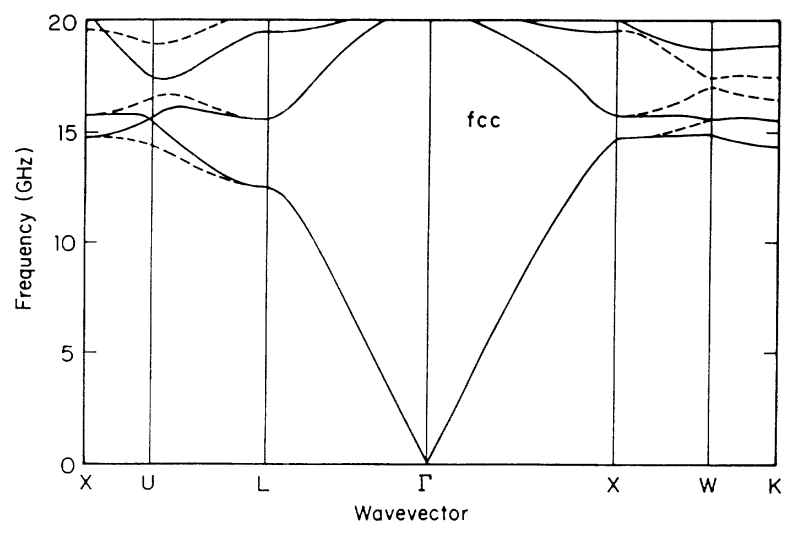

FIG. 1. Calculated photonic band structure along important symmetry lines in the Brillouin zone for a fcc dielectric structure composing of air spheres in a dielectric background of refractive index 3.5 . The filling ratio is $86 \%$ air and $14 \%$ dielectric material. Along $X-U-L$ and $X-W-K$, the dotted and solid lines indicate bands which couple only to $s$ and $p$ polarized light, respectively, in the experiment of Ref. 11.

and filling ratios for a fcc lattice of spheres. There is also a crossing of the second and third band along the symmetry line $U-X$ near $U$. These two features of the band structures prevent a gap from opening up in the fcc structure.

In the experiment, ${ }^{11}$ the photonic band structure was probed by measuring the transmission of EM waves incident on the (100) face of the "crystal." These waves are either $s$ polarized ( $\mathrm{E}$ parallel to the surface) or $p$ polarized ( $E$ in the incident plane). For wave vectors in the $X-U-L$ and the $X-W-K$ planes, reflection symmetry of the crystal causes a separation of the photon bands into one group which interacts only with $s$-polarized waves (indicated in Fig. 1 by dotted lines) and another group which interacts only with $p$-polarized waves (solid lines in Fig. 1). Along the $X-W$ line, the photonic gaps for $s$ polarization and $p$ polarization are identical at $X$ but as the wave vector moves towards $W$, the $s$ gap rises in frequency until at $W$ the bottom of the $s$ gap coincides with the top of the $p$ gap to give a degenerate state at $W$. Thus a gap will show up in experiments for $s$ and $p$ polarization individually. In the experiment, as the frequency of the EM wave increases, the transmission signal drops very sharply at the beginning of the gap so that the bottom of the gap is very well determined. At the $W$ point, there is good agreement between theory and experiment for the frequencies of the bottoms of the $s$ gap and the $p$ gap. ${ }^{17}$ However, the experiment seems to overestimate the frequency of the top of the gap compared with our calculations. Apparently, due to the finite crystal size, the experiment had inadequate resolution to observe the degeneracy of the bottom of the $s$ gap and the top of the $p$ gap at $W .^{17}$

Our analysis indicates that a photonic gap is inhibited in the simple fcc structure because of a symmetry-in- 


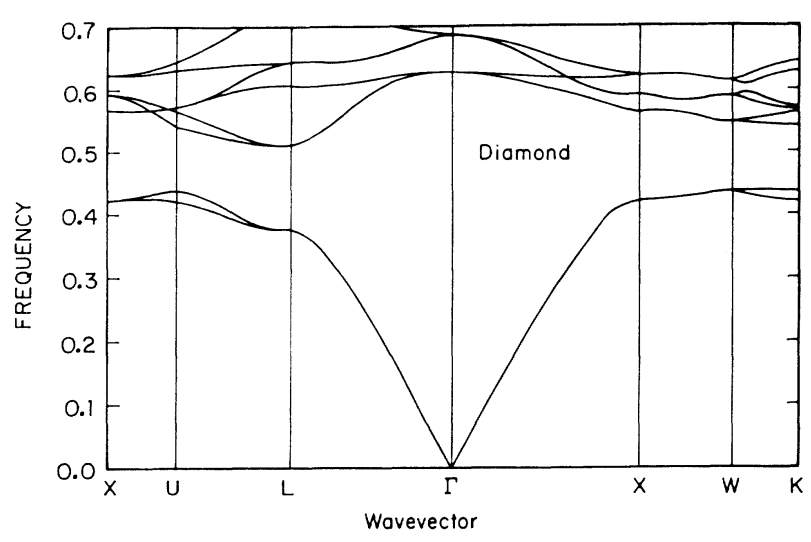

FIG. 2. Calculated photonic band structure for a diamond dielectric structure consisting of dielectric spheres of refractive index 3.6 in an air background. The filling ratio of the dielectric material is $34 \%$. The frequency is given in units of $c / a$, where $a$ is the cubic lattice constant of the diamond lattice.

duced degeneracy at the $W$ point. We, therefore, seek to change this degeneracy by changing the symmetry of the lattice. However, we would still like to keep the fcc Brillouin zone since it is most spherelike and favorable for the overlapping of band gaps at various Brillouin-zoneboundary wave vectors. The next structure we tried is the diamond lattice. We show in Fig. 2 an example for the case of a diamond lattice of dielectric spheres with a refractive index of 3.6 and a filling ratio of $34 \%$, i.e., when the spheres are just touching each other. We find that there exists a full photonic band gap in which EM waves are forbidden to propagate in any direction, including $W$ and $U$.

We have made a systematic examination of the photonic band structures for dielectric spheres and air spheres on a diamond lattice as a function of refractive index contrasts and filling ratios. In all the cases we examined, the lattice-constant $a$ was kept constant and the radius $r_{s}$ of the spheres was varied to change the filling fraction $f$. We found that when we fixed the refractive index at 3.6, photonic band gaps exist over a wide region of filling ratios for both dielectric spheres and air spheres. We plot in Fig. 3(a) the calculated size of the forbidden gap normalized to the midgap frequency for both cases. For dielectric spheres on a diamond lattice a maximum gap to midgap ratio $\left(\Delta \omega / \omega_{g}\right)$ of $15.7 \%$ is found at $f=37 \%$, whereas for the case of air spheres, $\Delta \omega / \omega_{g}$ can reach $28.8 \%$ at $f=81 \%$.

We notice that in the diamond structure, all the bands along the symmetry line from $W$ to $X$ are required to have twofold degeneracy. This favors the opening up of a gap between the second and third bands which is what is required for a photonic gap. We found that a band gap can be formed in the diamond structure at relatively low dielectric contrasts. We plot in Fig. 3(b) $\Delta \omega / \omega_{g}$ as a function of refractive-index contrast for a fixed dielectric
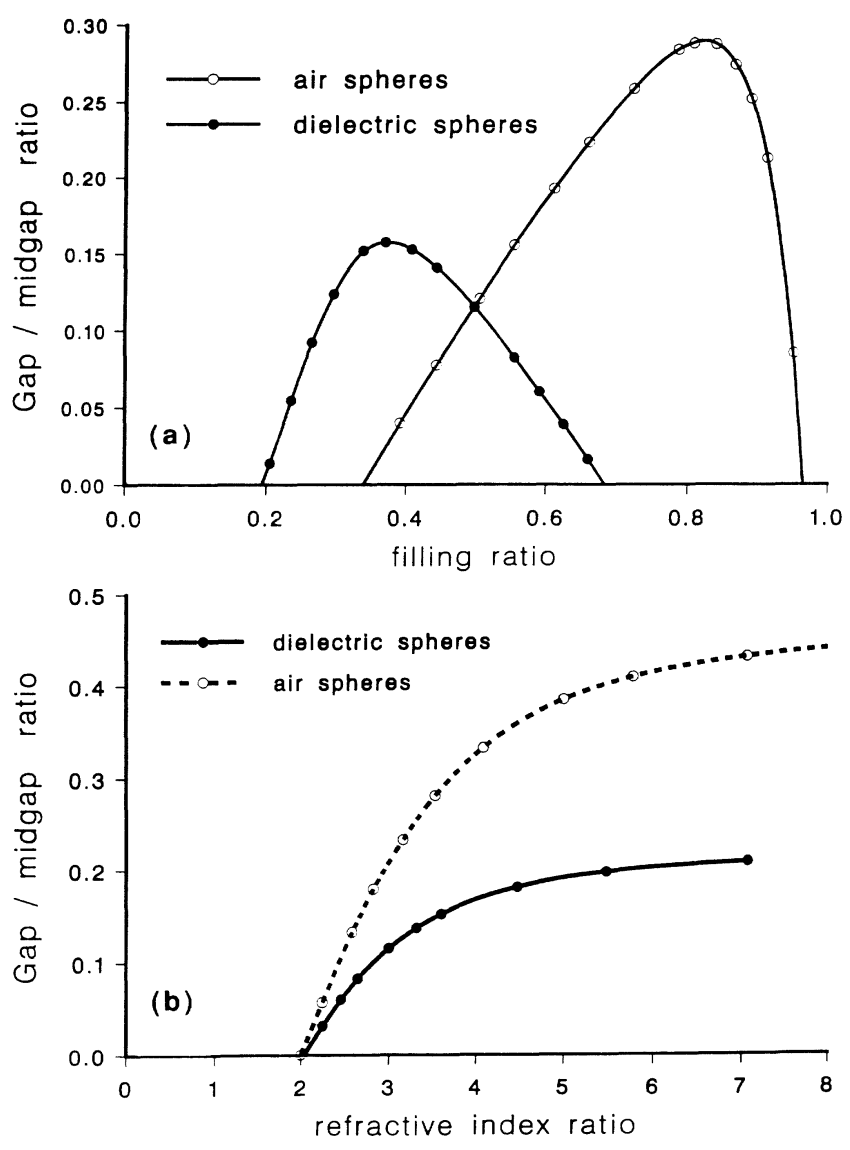

FIG. 3. (a) Gap to midgap frequency ratio $\left(\Delta \omega / \omega_{g}\right)$ as a function of filling ratio for the case of dielectric spheres in air and air spheres in dielectric. The refractive index of the material is chosen to be 3.6. (b) $\Delta \omega / \omega_{g}$ as a function of refractive index contrast for a fixed dielectric structure. The dotted line is for the case of air spheres in dielectric with a filling ratio of $81 \%$, and the solid line is for dielectric spheres in air with a filling ratio of $34 \%$.

structure. We keep $f=34 \%$ for the case of dielectric spheres in air and $f=81 \%$ for air spheres in a dielectric background. For both cases a photonic gap exists when the refractive-index contrast exceeds 2 . Such contrasts are easily accessible for visible light with existing dielectric materials. We observe that for increasing contrasts $\Delta \omega / \omega_{g}$ saturates to a value of $21 \%$ for the case of solid spheres and to a very large value of $46 \%$ for the case of air spheres.

In a number of previous theoretical investigations, it was asserted that solution of the Maxwell equations within the scalar-wave approximation can serve as a qualitative guide in determining the photonic band structure. We see in the present results that this is not true. In the fcc structure the scalar-wave approximation predicts the existence of a photonic gap whereas no gap exists in the exact solution. For the diamond lattice where the exact solution indicates a gap, the double degeneracy 
of the states along $W$ to $X$ prohibits the opening of a gap in the scalar approximation. Thus important symmetry information is lost when the vector nature of the EM field is neglected.

In conclusion, we have solved the problem of the propagation of physical EM waves in a three-dimensional periodic arrangement of dielectric structures. Our calculational scheme provides a very efficient way to obtain the crystal structure and the optimum parameters $\left(n_{a} / n_{b}\right.$ and $f$ ) for the maximum gap. We found that an arrangement of dielectric spheres in a diamond structure with easily obtainable dielectric constants clearly possesses a full photonic gap which can be verified in future experimental measurements. The traditional scalarwave approximation is found to be inadequate in describing important aspects of the photonic band problem.

We would like to thank E. Yablonovitch for invaluable discussions on the experimental data analysis and $\mathrm{E}$. $\mathrm{N}$. Economou for useful discussions. Ames Laboratory is operated by the U.S. Department of Energy by Iowa State University under Contract No. W-7405-Eng-82. This work was supported by the Director for Energy Research, Office of Basic Energy Sciences, including a grant of computer time on the Cray computer at the Lawrence Livermore Laboratory and by NATO Grant No. RG 769/87.

\footnotetext{
'For a recent review of the field, see Scattering and Localization of Classical Waves in Random Media, edited by $\mathrm{P}$. Sheng (World Scientific, Singapore, 1990).
}

${ }^{2}$ P. W. Anderson, Phys. Rev. 109, 1492 (1958); Philos. Mag. B 52, 5050 (1985).

${ }^{3}$ S. John, Phys. Rev. Lett. 53, 2169 (1984); Phys. Rev. B 31, 304 (1985).

${ }^{4}$ P. Sheng and Z. Q. Zhang, Phys. Rev. Lett. 57, 1879 (1986).

${ }^{5}$ K. Ayra, Z. B. Su, and J. L. Birman, Phys. Rev. Lett. 57, 2725 (1986).

${ }^{6}$ C. A. Condat and T. R. Kirkpatrick, Phys. Rev. Lett. 58, 226 (1987).

${ }^{7}$ C. M. Soukoulis, E. N. Economou, G. S. Grest, and M. H. Cohen, Phys. Rev. Lett. 62, 575 (1989); E. N. Economou and C. M. Soukoulis, Phys. Rev. B 40, 7977 (1989).

${ }^{8}$ S. John, Phys. Rev. Lett. 58, 2486 (1987); S. John and R. Rangavajan, Phys. Rev. B 38, 10101 (1988).

${ }^{9}$ E. N. Economou and A. Zdetsis, Phys. Rev. B 40, 1334 (1989).

${ }^{10}$ E. Yablonovitch, Phys. Rev. Lett. 58, 2059 (1987).

${ }^{11}$ E. Yablonovitch and T. J. Gmitter, Phys. Rev. Lett. 63, 1950 (1989); E. Yablonovitch, in Analogies in Optics and Micro Electronics, edited by W. van Haeringen and D. Lenstra (Kluwer Academic, The Netherlands, 1990), pp. 117-133.

${ }^{12}$ S. Satpathy, Ze Zhang, and M. R. Salehpour, Phys. Rev. Lett. 64, 1239 (1990); 65, 2478(E) (1990).

${ }^{13}$ K. M. Leung and Y. F. Liu, Phys. Rev. B 41, 10188 (1990); K. M. Ho, C. T. Chan, and C. M. Soukoulis (to be published)

${ }^{14}$ G. Kurizki and A. Z. Genack, Phys. Rev. Lett. 61, 2269 (1988).

${ }^{15}$ J. M. Drake and A. Z. Genack, Phys. Rev. Lett. 63, 259 (1989).

${ }^{16}$ This technique can also be adapted to the scalar-wave calculations, yielding a more efficient method than the one reported in Ref. 12. For details, see Ref. 13.

${ }^{17}$ E. Yablonovitch (private communication). 